\title{
Journal of Case Reports and Medical Images
}

Open Access | Review Article

\section{Pain in chemotherapy-induced peripheral neuropathy: NGF and the logic of topical phenytoin cream}

\author{
*Corresponding Author(s): Jan M Keppel Hesselink \\ Faculty of health, University of Witten-Herdecke, \\ Germany \\ Institute for Neuropathic Pain, The Netherlands \\ Email: jan@neuropathie.nu
}

Received: Aug 31, 2018

Accepted: Oct 12, 2018

Published Online: Oct 19, 2018

Journal: Journal of Case Reports and Medical Images

Publisher: MedDocs Publishers LLC

Online edition: http://meddocsonline.org/

Copyright: (C) Keppel Hesselink JM (2018). This Article is distributed under the terms of Creative Commons Attribution 4.0 International License

Keywords: Topical; Neuropathic; Treatment; Analgesia; Analgesic

\section{Introduction}

A great number of randomized clinical trials in pain in CIPN failed to demonstrate a significant treatment effect of various analgesics and co-analgesics [1-3]. One of the reasons recently proposed for this unfortunate finding is linked to the heterogeneity of the pathophysiology in CIPN. Sisignano et al (2014) pointed out that data from animal experiments indicate that the pathophysiological mechanisms of pain generation in CIPN are even more complex than those in painful diabetic neuropathy and herpes zoster neuropathy [4]. It is suggested that this heterogeneity of pain mechanisms, relates to the unsuccessful translation of preclinical findings to the treatment of pain

\begin{abstract}
In Chemotherapy-Induced Peripheral Neuropathy (CIPN) epidermal nerves and keratinocytes are damaged due to toxic influences of oncolytical therapies. One important detrimental factor recently described is the Nerve Growth Factor (NGF). NGF is produced by irritated keratinocytes, and NGF over production leads to over active nociceptors. This mechanism of peripheral sensitization induces localized peripheral pain. Both membranes of keratinocytes and nociceptors are rich in sodium channels. The broad acting sodium channel blocker phenytoin, applied in a suitable topical formulation, might decrease pain. In the past we reported a number of patients suffering from burning pain in both feet due to CINP, pain which did not subside after months or years following the chemotherapy. Topical treatment with phenytoin $10 \%$ diminished the pain considerably, and increased quality of life. We therefore postulate a close fit between phenytoin's mechanism of action and the pathophysiology of neuropathic pain in CIPN.
\end{abstract}

in CIPN. In the past we reported a number of patients suffering from burning pain in both feet due to CINP, pain which did not subside after months or years following the chemotherapy. Topical treatment with phenytoin $10 \%$ diminished the pain considerably, and increased quality of life [5-7]. It might be that an ex juvantibus approach using topical phenytoin,could result in relevant pain reduction in a subcohort of CIPN patients, in case of a close fit between phenytoin's mechanism of action and the pathophysiology of CIPN pain. We will briefly explore the theoretical background leading to this assumption. 


\section{CIPN: toxic damage of nociceptors}

CIPN can be induced by a number of neurotoxic anticancer compounds, such as the taxanes and platinum salts, Vinca alkaloids, and compounds such as thalidomide and bortezomib. Clearly these drugs damage nerve fibres, among which the unmyelinated fibers in the epidermis, the terminal nerve endings of either C or A $\delta$ fibers. Although skin biopsy is seen as the gold standard technique for diagnosing small fiber neuropathies, there are contradictory results on the relationship between skin biopsy data and neuropathic pain [8]. Thus, a diagnose-orientated approach in the treatment of pain in CINP will often fail due to the mismatch between the Mechanism Of Action (MOA) of the prescribed analgesic and the pathophysiological base of pain.

The quality of neuropathic pain in CINP is often described by qualifiers such as shooting and/or burning, and such pain qualities are reported in approximately $40 \%$ of patients with CIPN [9]. It is known that Vinca alkaloids and platinum salts have toxic effects on the DRG neurons, and increase the activity the voltage-dependent sodium channels [10-11]. This supports the rationale for the application of topical phenytoin, as we will discuss here under. The cell bodies of sensory first-order neurons which are located in the dorsal root ganglia extend into the dermis where they end and the nociceptors are located. Clearly neurotoxic effects can become manifest in the entire neuron, from its dendrites up to the last part of its axon in the epidermis. To build a case for topical treatment, we will focus on the epidermal structures and the part of the neuron ending in the epidermis, the nociceptors. This nociceptor and its adjacent tissue compartments, such as the keratinocytes and the immunecompetent cells are the drivers for peripheral sensitization and contribute to the experience if burning pain, as experienced in the skin.

\section{NGF as a key messenger molecule in peripheral sensitiza- tion}

One of the major inflammatory molecules playing a role in toxic damage of neurons is the Nerve Growth Factor (NGF). NGF upregulates voltage gated sodium channels expression in neuropathic pain states, and this is reported to be related to the sensitization of peripheral nociceptors [12]. Afferent neurons in neuropathic pain states demonstrate increased firing based on altered expression of several types of voltage-gated sodium channels [13]. Recently, increases in the expression of NGF in patients suffering from CINP has been demonstrated [14]. It was already in the last century, that the Nobel laureate Rita Levi-Montalcini discussed NGF as a pro-inflammatory agent, and pointed out the importance of modulating over active inflammations and pain states triggered by NGF [15]. More recently a pupil of Professor Levi-Montalcini, Dr. Luigi Aloe, and his colleagues pointed out that NGF is produced and released from the keratinocytes, in case of damage induced by toxins, and binds to receptors on the small nerve fibres [16].

Previously we discussed the importance of cross-talk between the keratinocytes and the nociceptors in localized peripheral neuropathic pain [17]. Already as early as 2004 it was found that in neuropathic patients with signs of hyperalgesia and allodynia, local increases of NGF levels were detectable, and it was suggested that anti-NGF agents could provide analgesia [18]. Topical phenytoin could be such an 'anti-NGF agent' and its use is supported by these findings.
Recently we demonstrated that it can be of use to apply topical formulations containing phenytoin, a broad acting sodium channel blocker, in pain due to CIPN. Phenytoin application resulted in a fast onset of action; within a time frame of $30 \mathrm{~min}$ utes, patients report clinical relevant reductions of pain [5-7]. This quick onset of action suggests indeed a local effect on the structures in the epidermis, such as the keratinocytes and the nociceptors (Figure 1). Moreover, we were not able to detect any levels of phenytoin in the blood of patients treated with $10 \%$ phenytoin cream [7].



Figure 1: Chemotherapy induces NGF production by keratinocytes; NGF stimulates the nociceptors to produce pain-signals: based on our case-studies, we postulate that while phenytoin blocks the sodium channels it can thus protect the nociceptors against the NGF influences, and might even reduce the NGF formation by the keratinocytes.

\section{Conclusion}

In the past we treated a number of patients suffering from pain due to CINP via the use of topical applied phenytoin $10 \%$ cream. In those cases, we reported in literature, clear analgesic effects emerged within $\mathbf{3 0}$ minutes after application. In the case of pain in CIPN, an ex juvantibus approach using topical administration of phenytoin cream is also supported by literature, especially since the inflammatory actions of NGF produced in the epidermal structures irritate the nociceptors. Of course, the pathophysiology of pain in CINP is complex, but in a subcohort of patients experiencing burning pain, an important pathophysiological mechanism is related to the over activity of the sodium channels on nociceptors and keratinocytes, linked to the over-expression of NGF. Such patients might be responders to a topical broad acting sodium channel blocker, as we have illustrated recently in a number of cases, reported in literature. [By using phenytoin and further characterize responders, the ex juvantibus approach might help to dissect the syndrome of pain in CIPN in pathophysiological uniform subgroups.

\section{Conflict of interest}

The author is patent holder of two patents related to the topical formulations of phenytoin in the treatment of pain: 1) Topicalphenytoin for the use in the treatment of peripheral neuropathic pain, and 2) Topical pharmaceutical composition containing phenytoinand a (co-) analgesic for the treatment of chronic pain. 


\section{References}

1. Finnerup NB, Sindrup SH, Jensen TS. The evidence for pharmacological treatment of neuropathic pain. PAIN. 2010; 150: 573581.

2. Hershman DL, Lacchetti C, Dworkin RH, Lavoie Smith EM, Bleeker $\mathrm{J}$, et al. Prevention and management of chemotherapy-induced peripheral neuropathy in survivors of adult cancers: American society of clinical oncology clinical practice guideline. J Clin Oncol. 2014; 32: 1941-67.

3. Rao RD, Michalak JC, Sloan JA, Loprinzi CL, Soori GS, et al. Efficacy of gabapentin in the management of chemotherapy-induced peripheral neuropathy: a phase 3 randomized, double-blind, placebo-controlled, crossover trial (NOOC3). Cancer. 2007; 110: 2110-2118.

4. Sisignano M, Baron R, Scholich K, Geisslinger G. Mechanismbased treatment for chemotherapy-induced peripheral neuropathic pain. Nat Rev Neurol. 2014; 10: 694-707.

5. Keppel Hesselink JM, Kopsky DJ. Fast onset of relief after topical phenytoin in neuropathic pain after chemotherapy. Trends Cancer Res Chemother. 2018; 1: 1-3.

6. Keppel Hesselink JM, Kopsky DJ. Pain in chemotherapy induced peripheral neuropathy treated with topical phenytoin cream. CP Cancer Sci. 2017; 1: 008.

7. Kopsky DJ, Keppel Hesselink JM. Phenytoin Cream for the Treatment of Neuropathic Pain: Case Series. Pharmaceuticals (Basel). 2018; 11: E53.

8. Truini A, Biasiotta A, Di Stefano G, Leone C, La Cesa S, et al. Does the epidermal nerve fibre density measured by skin biopsy in patients with peripheral neuropathies correlate with neuropathic pain? Pain. 2014; 155: 828-832

9. Geber C, Breimhorst M, Burbach B, Egenolf C, Baier B, et al. Pain in chemotherapy-induced neuropathy--more than neuropathic? Pain. 2013; 154: 2877-2887.
10. Park SB. Krishnan AV, Lin CS, Goldstein D, Friedlander M, et al. Mechanisms underlying chemotherapy-induced neurotoxicity and the potential for neuroprotective strategies. Curr. Med. Chem. 2008; 15: 3081-3094.

11. Nieto FR, Entrena JM, Cendán CM, Pozo ED, Vela JM, et al. Tetrodotoxin inhibits the development and expression of neuropathic pain induced by paclitaxel in mice. Pain 2008; 137: 520-531.

12. Siniscalco D, Giordano C, Rossi F, Maione S, de Novellis V. Role of neurotrophins in neuropathic pain. Role of neurotrophins in neuropathic pain. Curr Neuropharmacol. 2011; 9: 523-529.

13. Waxman SG, Zamponi GW. Regulating excitability of peripheral afferents: emerging ion channel targets. Nat Neurosci. 2014; 17: 153-163.

14. Velasco R, Navarro X, Gil-Gil M, Herrando-Grabulosa M, Calls A, et al. Neuropathic Pain and Nerve Growth Factor in Chemotherapy-Induced Peripheral Neuropathy: Prospective Clinical-Pathological Study. J Pain Symptom Manage. 2017; 54: 815-825.

15. Keppel Hesselink J. Professor Rita Levi-Montalcini on Nerve Growth Factor, Mast Cells and Palmitoylethanolamide, an Endogenous Anti-Inflammatory and Analgesic Compound. J Pain Relief. 2013; 2: 114.

16. Aloe L, Rocco ML, Bianchi P, Manni L. Nerve growth factor: from the early discoveries to the potential clinical use. J Transl Med. 2012; 10: 239.

17. Keppel Hesselink JM, Kopsky DJ, AK Bhaskar. Skin matters! The role of keratinocytes in nociception: A rational argument for the development of topical analgesics. J Pain Res. 2017; 10: 1-8.

18. Anand P. Neurotrophic factors and their receptors in human sensory neuropathies.Prog Brain Res. 2004; 146: 477-492. 